\title{
The Optimal Rubbling Number of Paths, Cycles, and Grids
}

\author{
Zheng-Jiang Xia $\mathbb{i}$ and Zhen-Mu Hong $\mathbb{1}$ \\ School of Finance, Anhui University of Finance \& Economics, Bengbu 233030, China \\ Correspondence should be addressed to Zheng-Jiang Xia; xzj@mail.ustc.edu.cn
}

Received 30 January 2021; Revised 28 February 2021; Accepted 2 April 2021; Published 5 July 2021

Academic Editor: M. Irfan Uddin

Copyright (c) 2021 Zheng-Jiang Xia and Zhen-Mu Hong. This is an open access article distributed under the Creative Commons Attribution License, which permits unrestricted use, distribution, and reproduction in any medium, provided the original work is properly cited.

\begin{abstract}
A pebbling move on a graph $G$ consists of the removal of two pebbles from one vertex and the placement of one pebble on an adjacent vertex. Rubbling is a version of pebbling where an additional move is allowed, which is also called the strict rubbling move. In this new move, one pebble each is removed from $u$ and $v$ adjacent to a vertex $w$, and one pebble is added on $w$. The rubbling number of a graph $G$ is the smallest number $m$, such that one pebble can be moved to each vertex from every distribution with $m$ pebbles. The optimal rubbling number of a graph $G$ is the smallest number $m$, such that one pebble can be moved to each vertex from some distribution with $m$ pebbles. In this paper, we give short proofs to determine the rubbling number of cycles and the optimal rubbling number of paths, cycles, and the grid $P_{2} \times P_{n}$; moreover, we give an upper bound of the optimal rubbling number of $P_{m} \times P_{n}$.
\end{abstract}

\section{Introduction}

Pebbling in graphs was first introduced by Chung [1]. It has its origin in number theory and also can be viewed as a model for the transportation of resources, starting from a pebble distribution on the vertices of a connected graph.

Let $G$ be a simple connected graph; we use $V(G)$ and $E(G)$ to denote the vertex set and edge set of $G$, respectively. $d(u, v)$ is the distance of $u$ and $v$, and we write $u \sim v$ if they are adjacent. $N(v)=\{u \mid u \sim v\}$ is the neighbour of $v$, and $d(v)=|N(v)|$ is the degree of $v$. Let $H$ be a subgraph of $G$, and we use $d_{H}(v)$ to denote the degree of $v$ in $H$. A pebble distribution $D$ on $G$ is a function $D: V(G) \longrightarrow N(N$ is the set of nonnegative integers), where $D(v)$ is the number of pebbles on $v$ and $|D|=\sum_{v \in V(D)} D(v)$ is the size of $D$.

A pebbling move consists of the removal of two pebbles from a vertex and the placement of one pebble on an adjacent vertex. For two pebble distributions $D$ and $D^{\prime}$ of $G$, we say that $D$ contains $D^{\prime}$ if $D(v) \geq D^{\prime}(v)$ for all $v \in V(G)$; and $D^{\prime}$ is reachable from $D$ if we can obtain a distribution which contains $D^{\prime}$ from $D$ by pebbling moves. For a graph $G$ and a vertex $v$, we call $v$ a root (or target vertex) if the goal is to place pebbles on $v$. If $t$ pebbles can be moved to $v$ from $D$ by a sequence of pebbling moves, then we say that $D$ is $t$-fold $v$-solvable, and $v$ is t-reachable from $D$. If $D$ is $t$-fold $v$-solvable for every vertex $v$, we say that $D$ is $t$-solvable. Let $S$ be a subset of $V(G)$. If $D$ is $v$-solvable for all $v \in S$, then we say $D$ is $S$-solvable.

The t-pebbling number of a graph $G$, denoted by $f_{t}(G)$, is the smallest number $m$, such that every distribution with size $m$ is $t$-solvable. While $t=1$, we use $f(G)$ instead of $f_{1}(G)$, which is called the pebbling number of graph $G$. The optimal pebbling number $f_{\text {opt }}(G)$ of a graph $G$ is the minimum number $m$ such that there exists a solvable distribution with size $m$.

Rubbling is a version of pebbling where an additional move is allowed, which is also called the strict rubbling move. In this new move, one pebble each is removed from $u$ and $v$ adjacent to a vertex $w$, and one pebble is added on $w$. The t-rubbling number of a graph $G$, denoted by $\rho_{t}(G)$, is the smallest number $m$, such that every distribution with size $m$ is $t$-solvable by rubbling moves. Similarly, while $t=1$, we use $\rho(G)$ instead of $\rho_{1}(G)$, which is called the rubbling number of graph $G$. The optimal rubbling number $\rho_{\text {opt }}(G)$ of a graph $G$ is the minimum number $m$ such that there exists a solvable distribution with size $m$ by rubbling moves. 
There are many papers about pebbling on graphs; one can view the survey paper [2] written by Hurlbert. Rubbling is a new parameter with few results. The basic theory about rubbling and optimal rubbling was developed by Belford and Sieben [3]; they determined the rubbling numbers of trees and cycles, the optimal rubbling numbers of paths and cycles, and so on. The rubbling numbers of complete $m$-ary trees are studied in [4], the rubbling numbers of caterpillars are given in [5], the optimal rubbling numbers of ladders, prisms, and Möbius ladders are determined in [6], and in [7], they give the bounds for the rubbling numbers of diameter 2 graphs.

In this paper, we give some new proofs to determine the rubbling numbers of cycles and the optimal rubbling numbers of paths, cycles, ladders, prisms, and Möbius ladders. Also, we give an upper bound of the optimal rubbling number of $P_{3} \times P_{n}$, from which we also get an upper bound of the optimal rubbling number of $P_{m} \times P_{n}$.

\section{Main Result}

Assume that $v, w \in N(u)$. A pebbling move from $v$ to $u$ is the removal of two pebbles from $v$ and addition of one pebble on $u$, denoted by $(v, v \longrightarrow u)$; a rubbling move from $\{v, w\}$ to $u$ is the removal of one pebble from $v$ and one pebble from $w$ and addition of one pebble on $u$, denoted by $(v, w \longrightarrow u)$.

The following lemma holds clearly.

Lemma 1 (see [3]). $2^{d(G)} \leq \rho(G) \leq f(G)$.

Definition 1 (see [3]). Given a multiset $S$ of rubbling moves on $G$, the transition digraph $T(G, S)$ is a directed multigraph whose vertex set is $V(G)$, and each move $(v, w \longrightarrow u)$ in $S$ isrepresented by two directed edges $(v, u)$ and $(w, u)$. The transition digraph of a rubbling sequence $S=\left(S_{1}, \ldots, s_{n}\right)$ is $T(G, s)=T(G, S)$, where $S=\left\{s_{1}, \ldots, s_{n}\right\}$ is the multiset of moves in $s$.

Lemma 2 (see $[3,8]$, no-cycle lemma). Let $S$ be a sequence of rubbling moves on $G$, reaching a distribution $D$. Then, there exists a sequence $S^{*}$ of rubbling moves, reaching a distribution $D^{*}$, such that

(1) On each vertex $v, D^{*}(v) \geq D(v)$.

(2) $T\left(G, S^{*}\right)$ does not contain any directed cycles.

A thread in a graph is a path containing vertices of degree 2. By Lemma 2, we can get the following.

Lemma 3 (see [3]). Let $P$ be a thread in $G$; if vertex $x \notin V(P)$ is t-reachable from the pebble distribution $D$ by a sequence of rubbling moves, then $x$ is t-reachable from a rubbling sequence in which there is no strict rubbling move of the form $(v, w \longrightarrow u)$ for $u \in V(P)$.

Definition 2 (see [9]). For a given distribution $D$ on $V(G)$, assume $d(v)=2$ and $D(v) \geq 3$. A smoothing move from $v$ changes $D$ by removing two pebbles from $v$ and adding one pebble on each neighbour of $v$.
Lemma 4 (see [9]). Let $D$ be a distribution on a graph $G$ with distinct vertices $u$ and $v$, where $d(v)=2, D(v) \geq 3$. If $u$ is $t$-reachable under $D$, then $u$ is $t$-reachable under the distribution $D^{\prime}$ obtained by making a smoothing move from $v$.

A distribution is smooth, if it has at most two pebbles on every vertex with degree 2 . A vertex $v$ is unoccupied under $D$ if $D(v)=0$.

Lemma 5 (smoothing lemma, [9]). If $G$ is a connected $n$-vertex graph, with $n \geq 3$, then $G$ has a smooth optimal distribution with all leaves unoccupied.

Lemma 6 (see [9]). In a path with a smooth distribution D having at most two pebbles on each endpoint, let $v$ be an unoccupied vertex. If $v$ is an endpoint, then $v$ is not 2reachable under $D$. If $v$ is an inner vertex, then no pebble can be moved out from $v$ without using an edge in both directions.

Definition 3 (see [9]). A graph $H$ is a quotient of a graph $G$ if the vertices of $H$ correspond to the sets in a partition of $V(G)$, and distinct vertices of $H$ are adjacent if at least one edge of $G$ has endpoints in the sets corresponding to both vertices of $H$. In other words, each set in the partition of $V(G)$ collapses to a single vertex of $H$. If $H$ is a quotient of $G$ via the surjective map $\phi: V(G) \longrightarrow V(H)$ and $D$ is a distribution on $G$, then the quotient distribution $D^{*}$ is the distribution on $H$ defined by $D^{*}(u)=\sum_{v \in \phi^{-1}(u)} D(v)$.

Lemma 7 (collapsing lemma, [9]). Let $H$ be a quotient of $G$ and $D^{*}$ be the quotient distribution in $H$. Then, $D$ is $t$-fold $v$-solvable by a sequence of pebbling moves $\Rightarrow D^{*}$ is $t$-fold $\phi(v)$-solvable by a sequence of pebbling moves. In particular, $f_{\text {opt }}(G) \geq f_{\text {opt }}(H)$.

Similarly, we can get the collapsing lemma on rubbling.

Lemma 8 Let $H$ be a quotient of $G$ and $D^{*}$ be the quotient distribution on $H$. Then, $D$ is $t$-fold $v$-solvable by a sequence of rubbling moves $\Rightarrow D^{*}$ is $t$-fold $\phi(v)$-solvable by a sequence of rubbling moves. In particular, $\rho_{\text {opt }}(G) \geq \rho_{\text {opt }}(H)$.

Proof. The proof is similar to the proof of Lemma 7 in [9].

\subsection{Rubbling and Optimal Rubbling in Paths and Cycles}

Lemma 9 (see $[10,11])$. The t-pebbling numbers of the cycles $C_{2 n+1}$ and $C_{2 n}$ are

$$
\begin{aligned}
f_{t}\left(C_{2 n+1}\right) & =2\left\lfloor\frac{2^{n+1}}{3}\right\rfloor+(t-1) 2^{n}+1, \\
f_{t}\left(C_{2 n}\right) & =2^{n} t .
\end{aligned}
$$

In this section, we give short proofs about the rubbling number of cycles and the optimal rubbling numbers of paths and cycles, which are determined in [3].

Theorem 1 (see [3]). The rubbling numbers of cycles are 


$$
\begin{aligned}
\rho\left(C_{2 n}\right) & =2^{n} \\
\rho\left(C_{2 n+1}\right) & =\left\lfloor\frac{7 \cdot 2^{n-1}-2}{3}\right\rfloor+1 .
\end{aligned}
$$

Proof. For the even cycle $C_{2 n}$, by Lemma 1, we have $2^{n} \leq \rho\left(C_{2 n}\right) \leq f\left(C_{2 n}\right)=2^{n}$, over.

For the odd cycle $C_{2 n+1}$, assume the target vertex is $v_{0}$ which is adjacent to $v_{1}$ and $v_{-1}$. Let $D$ be a pebble distribution on $C_{2 n+1}$, and $D\left(v_{0}\right)=0$. We collapse the vertices $\left\{v_{-1}, v_{0}, v_{1}\right\}$ into one vertex $x$ to get a new graph $C_{2 n-1}$ and the quotient distribution $D^{*}$ on $C_{2 n-1}$, with $D^{*}(x)=D\left(v_{-1}\right)+D\left(v_{0}\right)+D\left(v_{1}\right)$ and $D^{*}(y)=D(y)$ for $y \neq x$.

By Lemma 3, we may assume that there is no strict rubbling move of the form $(v, w \longrightarrow u)$ where $u \neq v_{0}$. So, one pebble can be moved to $v_{0}$ from $D$ on $C_{2 n+1}$ by a sequence of rubbling moves $\Leftrightarrow$ two pebbles can be moved to $x$ from $D^{*}$ on $C_{2 n-1}$ by a sequence of pebbling moves. Thus, $\rho\left(C_{2 n+1}\right)=f_{2}\left(C_{2 n-1}\right)=2\left\lfloor 2^{n} / 3\right\rfloor+2^{n-1}+1$ (the last equality holds from Lemma 9). A simple calculation can show that $2\left\lfloor 2^{n} / 3\right\rfloor+2^{n-1}+1=\left\lfloor\left(7 \cdot 2^{n-1}-2\right) / 3\right\rfloor+1$.

Lemma 10 (see [9]). The optimal pebbling numbers of paths and cycles are

$$
f_{\text {opt }}\left(P_{n}\right)=f_{\text {opt }}\left(C_{n}\right)=\left\lceil\frac{2 n}{3}\right\rceil .
$$

Moreover, we can get the following lemma.

Lemma 11. Let $D$ be a pebble distribution with $2 k$ pebbles on $P_{3 k}=v_{1}, \ldots, v_{3 k}$. If $D$ is solvable by pebbling moves, then $D\left(v_{3 i+2}\right)=2$ for $0 \leq i \leq k-1$, and $D(v)=0$ otherwise.

Proof. We show it by induction on $k$; it holds for $k=1$.

We can move $\left\lfloor D\left(v_{1}\right) / 2\right\rfloor$ pebbles from $v_{1}$ to $P_{3 k-1}=v_{2}, \ldots, v_{3 k}$, so by induction, $2 k-D\left(v_{1}\right)+\left\lfloor D\left(v_{1}\right) / 2\right\rfloor \geq f_{\text {opt }}\left(P_{3 k-1}\right)=2 k$, and thus $D\left(v_{1}\right)=0$. Similarly, $D\left(v_{3 k}\right)=0$.

We make smoothing moves on $P_{3 k}$ from $D$ to obtain a smooth pebble distribution $D^{*}$.

Since $|D|=2 k<n=3 k$, there exist many vertices unoccupied under $D^{*}$. Assume $v_{i}$ is a vertex unoccupied under $D^{*}$. Let $L_{1}=v_{1}, \ldots, v_{i}$ and $L_{2}=v_{i+1}, \ldots, v_{3 k}$. By Lemma 6 , we cannot move two pebbles to $v_{i}$ using only one direction.

If $v_{i}$ is solvable by $\left(v_{i-1}, v_{i-1} \longrightarrow v_{i}\right)$, then we can get that $\left.D^{*}\right|_{L_{i}}$ is $L_{i}$-solvable, and thus $\left|D^{*}\right| \geq f_{\text {opt }}\left(P_{i}\right)+f_{\text {opt }}\left(P_{3 k-i}\right)$. Assume $i=3 j+r$ for $0 \leq r \leq 2$. If $r=0$, then $\left|D^{*}\right| \geq 2 j+2(k-j)=2 k$. If $r=1$, then $\left|D^{*}\right| \geq 2 j+$ $1+2(k-j)=2 k+1$, a contradiction to $|D|=2 k$. If $r=2$, then $\left|D^{*}\right| \geq 2 j+2+2(k-j-1)+1=2 k+1$, a contradiction to $|D|=2 k$, and thus $i=3 j$.

By a similar argument, if $v_{i}$ is solvable by $\left(v_{i+1}\right.$, $v_{i+1} \longrightarrow v_{i}$ ), then we can get $i=3 j+1$.

Thus, we can always partition the path $P_{3 k}$ into two paths $L_{1}=v_{1}, \ldots, v_{3 j}$ and $L_{2}=v_{3 j+1}, \ldots, v_{3 k}$, and $\left.D^{*}\right|_{L_{i}}$ is solvable in $L_{i}$ for $i=1,2$, respectively.
By induction, we know that $D^{*}\left(v_{3 i+2}\right)=2$ for $0 \leq i \leq k-1$, and $D^{*}(v)=0$ otherwise.

Note that making a smooth move on the vertex $v$ leaves at least one pebble on $v$, which means that if we can make a smooth move under $D$, then at least two adjacent vertices have pebbles under $D^{*}$, a contradiction to $D^{*}\left(v_{3 i+2}\right)=2$, and $D^{*}(v)=0$ otherwise. So, $D=D^{*}$, which completes the proof.

In [3], Belford and Sieben determined the optimal rubbling number of paths and cycles; here we give a short proof.

Theorem 2 (see [3]). The optimal rubbling number of path is $\rho_{\text {opt }}\left(P_{n}\right)=\lceil n+1 / 2\rceil$, and the optimal rubbling number of cycle is $\rho_{\text {opt }}\left(C_{n}\right)=\lceil n / 2\rceil$ for $n \geq 3$.

Proof

Upper Bound. For $P_{n}=v_{1}, v_{2}, \ldots, v_{n}$, let $D$ be a distribution so that $D\left(v_{i}\right)=1$ for $i$ is odd or $i=n$, and $D\left(v_{i}\right)=0$ otherwise; clearly $D$ is solvable, and $|D|=\lceil n+1 / 2\rceil$. For $C_{n}=v_{1}, v_{2}, \ldots, v_{n}$, let $D$ be a distribution so that $D\left(v_{i}\right)=1$ for $i$ is odd, and $D\left(v_{i}\right)=$ 0 otherwise; clearly $D$ is solvable, and $|D|=\lceil n / 2\rceil$.

Lower Bound. First we show it for the path $P_{n}$. We use induction on $n$. By a simple calculation, one can show that it holds for $n \leq 3$.

If $n>3$, assume it holds for $P_{j}$ for all $j<n$. From the upper bound and Lemma 10, we know that $\rho_{\text {opt }}\left(P_{n}\right)<f_{\text {opt }}\left(P_{n}\right)$. Let $D$ be a solvable pebble distribution with $\rho_{\text {opt }}\left(P_{n}\right)$ pebbles on $P_{n}$; there must exist a vertex $v_{i}$, which is reachable only by the strict rubbling move $\left(v_{i-1}, v_{i+1} \longrightarrow v_{i}\right)$. Let $P_{1}=v_{1}, \ldots, v_{i-1}, P_{2}=v_{i+1}, \ldots, v_{n}$ be two subpaths of $P_{n}$; then, by Lemma 3, we do not need the strict rubbling move $\left(v_{i-1}, v_{i+1} \longrightarrow v_{i}\right)$ to solve the vertices in $P_{i}$ for $i=1,2$. That means $\left.D\right|_{P_{i}}$ is $v$-solvable for all $v \in P_{i}$ $(i=1,2)$, so $\quad|D|_{P_{i}} \mid \geq \rho_{\text {opt }}\left(P_{i}\right) \quad$ for $\quad i=1,2$. So, $|D|=\left.|D|_{P_{1}}|+| D\right|_{P_{2}} \mid \geq\lceil i / 2\rceil+\lceil n-i+1 / 2\rceil \geq\lceil n+1 / 2\rceil$.

Now we show it for the cycle $C_{n}$; similarly, by a simple calculation, one can show that it holds for $n=3$.

If $n>3$, from the upper bound and Lemma 10, we know that $\rho_{\text {opt }}\left(C_{n}\right)<f_{\text {opt }}\left(C_{n}\right)$. Thus, let $D$ be an optimal pebble distribution with $\rho_{\text {opt }}\left(C_{n}\right)$ pebbles on $C_{n}$, and there must exist a vertex $v_{i}$, which is reachable only by a strict rubbling move $\left(v_{i-1}, v_{i+1} \longrightarrow v_{i}\right)$. Then, by Lemma 3 , we do not need the strict rubbling move $\left(v_{i-1}, v_{i+1} \longrightarrow v_{i}\right)$ to solve the vertices in $C_{n} \backslash v_{i}$, and thus $D$ is solvable on $P_{n-1}=C_{n} \backslash v_{i}$, so $|D| \geq \rho_{\text {opt }}\left(P_{n-1}\right)=\lceil n / 2\rceil$, which completes the proof.

\section{Optimal Rubbling in Ladders and Prisms}

Let $G$ and $H$ be simple connected graphs; we define the Cartesian product $G \times H$ to be the graph with vertex set $V(G \times H)$ and edge set the union of $\{(a v, b v) \mid(a, b) \in E(G), v \in E(H)\}$ and $\{(u x, u y) \mid u \in V(G)$, $(x, y) \in E(H)\}$. We call $P_{n} \times P_{2}$ a ladder and $C_{n} \times P_{2}$ a prism. It is clear that a prism can be obtained from a ladder by joining the 4 endvertices by two edges to form two vertexdisjoint $C_{n}$ subgraphs. If the four endvertices are joined by 
two new edges in a switched way to get a $C_{2 n}$ subgraph, then a Möbius ladder $M_{n}$ is obtained.

The optimal rubbling numbers of ladders, prisms, and Möbius ladders are determined in [6]; here we give new proofs of these results.

Let $P_{n}=v_{1}, v_{2}, \ldots, v_{n}$ and $P_{2}=x y$. The vertices of $P_{n} \times$ $P_{2}$ are denoted by $v_{i} x$ and $v_{i} y$ for $1 \leq i \leq n$. Let $H_{n}=\left(P_{n+1} \times P_{2}\right) \backslash v_{n+1} x$. Then, we have the following.

Theorem 3. Let $n=3 k+r, 0 \leq r \leq 2$; the optimal rubbling number of the ladder $P_{n} \times P_{2}$ [6] is

$$
\rho_{\text {opt }}\left(P_{3 k+r} \times P_{2}\right)= \begin{cases}2 k+1 & \text { if } r=0 \\ 2 k+2 & \text { if } r=1=\left\lceil\frac{2(n+1)}{3}\right\rceil . \\ 2 k+2 & \text { if } r=2\end{cases}
$$

The optimal rubbling number of $H_{n}$ is

$$
\rho\left(H_{n}\right)=\rho\left(P_{n-1} \times P_{2}\right)+1 .
$$

Proof

Upper Bound. Let $D$ be a distribution on $P_{n} \times P_{2}$ or $H_{n} \times P_{2}$.

If $r=0$, then $D\left(v_{i} x\right)=1$ for $i \equiv 1(\bmod 3) ; D\left(v_{i} y\right)=1$ for $i \equiv 2(\bmod 3)$ and $i \neq n-1 ; D\left(v_{n} y\right)=2$ and $D(v)=$ 0 otherwise.

If $r=1$, then $D\left(v_{i} x\right)=1$ for $i \equiv 1(\bmod 3)$ and $i \neq n$; $D\left(v_{i} y\right)=1$ for $i \equiv 2(\bmod 3) ; D\left(v_{n} y\right)=2$ and $D(v)=0$ otherwise.

If $r=2$, for $P_{n} \times P_{2}$, then $D\left(v_{i} x\right)=1$ for $i \equiv 1(\bmod 3)$; $D\left(v_{i} y\right)=1$ for $i \equiv 2(\bmod 3) ; D(v)=0$ otherwise. For $H_{n}$, we add one more pebble on $v_{n+1} y$.

One can check that $D$ is solvable for all $P_{n} \times P_{2}$ and $H_{n}$. Lower Bound. Note that the diameter of $P_{n} \times P_{2}$ is $n$, and let $R_{i}$ be the set of vertices with distance $i$ from $v_{1} x$ $(1 \leq i \leq n), R_{0}=v_{1} x$; if we collapse $R_{i}$ into one vertex $r_{i}$ for $0 \leq i \leq n$, then we can get a path $\widetilde{P}_{n+1}=r_{0}, \ldots, r_{n}$ (see Figure 1). By Lemma 10, the optimal pebbling number of $\widetilde{P}_{n+1}$ is $f_{\text {opt }}\left(\widetilde{P}_{n+1}\right)=\lceil 2(n+1) / 3\rceil$. By Lemma $8, \rho_{\text {opt }}\left(H_{n}\right) \geq \rho_{\text {opt }}\left(P_{n} \times P_{2}\right)$. So, we only need to show that $\rho_{\text {opt }}\left(P_{n} \times P_{2}\right) \geq f_{\text {opt }}\left(\widetilde{P}_{n+1}\right)$ and $\rho_{\text {opt }}\left(H_{3 k+2}\right) \geq 2 k+3$.

We use induction on $n$ for both $H_{n}$ (only need to show while $n \equiv 2(\bmod 3))$ and $P_{n} \times P_{2}$; it holds for $n \leq 2$ clearly.

Assume it holds for $h<n$.

Case 1. First we consider $P_{n} \times P_{2}$, and let $D$ be a solvable distribution on $P_{n} \times P_{2}$ with $\rho_{\text {opt }}\left(P_{n} \times P_{2}\right)$ pebbles; then, we collapse $R_{i}$ into one vertex $r_{i}$ for $0 \leq i \leq n$ to get a path $\widetilde{P}_{n+1}$ with length $n$ and the quotient distribution $D^{*}$ on $\widetilde{P}_{n+1}$. By Lemma $8, D^{*}$ is solvable in $\widetilde{P}_{n+1}$. We will show that $\rho_{\text {opt }}\left(P_{n} \times P_{2}\right) \geq f_{\text {opt }}\left(\widetilde{P}_{n+1}\right)$.

Assume $\rho_{\text {opt }}\left(P_{n} \times P_{2}\right)<f_{\text {opt }}\left(\widetilde{P}_{n+1}\right)$; since $D^{*}$ is a solvable distribution on $\widetilde{P}_{n+1}$, there must exist some $r_{i}$ of $\widetilde{P}_{n+1}$ which can be reachable only by the strict rubbling move

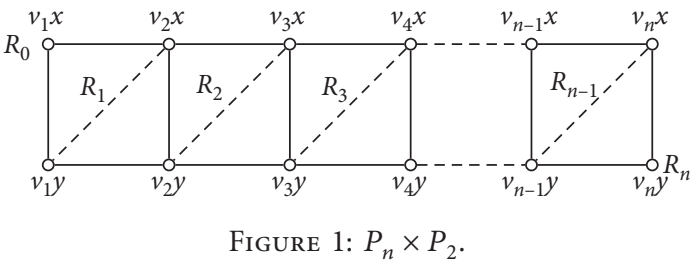

$\left(r_{i-1}, r_{i+1} \longrightarrow r_{i}\right)$. By Lemmas 3 and 8 , we claim the following.

Claim 1. At most one pebble can be moved to $r_{i-1}\left(r_{i+1}\right)$ under $D^{*}$ and at most one pebble can be moved to $R_{i-1}\left(R_{i+1}\right)$ under $D$.

Let $Q_{1}=r_{0}, \ldots, r_{i-1}, Q_{2}=r_{i+1}, \ldots, r_{n}$ be two subpaths of $\widetilde{P}_{n+1}$ and $W_{1}$ and $W_{2}$ be the subgraphs of $P_{n} \times P_{2}$ which induce $Q_{1}$ and $Q_{2}$, respectively.

Now we will show that $|D|_{W_{j}} \mid \geq \rho_{\text {opt }}\left(W_{j}\right)$ for $j=1,2$. Note that $W_{1}$ is isomorphic to $H_{i-1}$ and $W_{2}$ is isomorphic to $H_{n-i-1}$.

Notation. For simplicity, a rubbling move $\left(R_{i-1}, R_{i+1} \longrightarrow R_{i}\right)$ in $P_{n} \times P_{2}$ means a rubbling move $(v, u \longrightarrow w)$ for some $v \in R_{i-1}, u \in R_{i+1}$ and $w \in R_{i}$. And similarly, we can define a rubbling move $\left(R_{i-1}, u \longrightarrow R_{i}\right)$ and so on.

If $\left.D\right|_{W_{j}}$ is $W_{j}$-solvable, then $|D|_{W_{j}} \mid \geq \rho_{\text {opt }}\left(W_{j}\right)$, and we are done. Assume $\left.D\right|_{W_{j}}$ is not $W_{j}$-solvable for some $j$; without loss of generality, assume $\left.D\right|_{W_{2}}$ is not $W_{2}$-solvable. If $i=n-1$, then $W_{2}=\left\{v_{n} y\right\}$, and the strict rubbling move $\left(r_{n-2}, r_{n} \longrightarrow r_{n-1}\right)$ means that $D^{*}\left(r_{n}\right) \geq 1$, which means $D\left(v_{n} y\right) \geq 1$, a contradiction to $\left.D\right|_{W_{2}}$, is not $W_{2}$-solvable. Thus, $i<n-1$.

Since $\left.D\right|_{W_{2}}$ is not $W_{2}$-solvable, to solve some vertex of $W_{2}$, we must use the pebbles on $W_{1}$. Note that we can move at most one pebble on $R_{i-1}$ from $\left.D\right|_{W_{1}}$, and we must use the strict rubbling move $\left(R_{i-1}, R_{i+1} \longrightarrow R_{i}\right)$ under $D$.

Consider the vertex $v_{i} y \in R_{i}, N\left(v_{i} y\right)=R_{i-1} \cup\left\{v_{i+1} y\right\}$. Since $v_{i} y$ is reachable from $D$, we can move two pebbles to $N\left(v_{i} y\right)$. From Claim 1, we can get that we can move one pebble to $R_{i-1}$ and one pebble to $v_{i+1} y$ at the same time. Thus, $v_{i+1} y$ is reachable under $\left.D\right|_{W_{2}}$.

Subcase 1. $D\left(v_{i+1} y\right)=0$. Then, we can move two pebbles on $v_{i+2} y$ under $\left.D\right|_{W_{2}}$ (for $v_{i+1} y$ is just adjacent to one vertex $v_{i+2} y$ of $\left.W_{2}\right)$. So, $v_{i+2} x$ is reachable under $\left.D\right|_{W_{2}}$, too.

Note that the pebble moved on $R_{i}$ cost at least two pebbles on $v_{i+2} y$ and we can move at most one pebble to $R_{i+1}$ by using this pebble. We can use $\left(R_{i+2}, R_{i+2} \longrightarrow R_{i+1}\right)$ instead of the rubbling sequence which contains the strict rubbling move $\left(R_{i-1}, R_{i+1} \longrightarrow R_{i}\right)$ and aim to move one pebble to $R_{i+1}$, so $\left.D\right|_{W_{2}}$ is $W_{2}$-solvable.

Subcase 2. $D\left(v_{i+1} y\right)=1$. Then, at most one pebble can be moved to $R_{i+2}$ under $\left.D\right|_{W_{2}}$; otherwise, one more pebble can be moved from $r_{i+2}$ to $r_{i+1}$, and then there are two pebbles on $r_{i+1}$, and one pebble can be moved to $r_{i}$ under $D^{*}$, a contradiction. 
By a similar argument, we can show there exists some vertex in $W_{2}$ so that we must use the strict rubbling move $\left(R_{i-1}, v_{i+1} y \longrightarrow v_{i+1} x\right)$ to solve this vertex, and the next rubbling move must be $\left(R_{i+2}, v_{i+1} x \longrightarrow v_{i+2} x\right)$. Since at most one pebble can be moved to $R_{i+2}$ under $\left.D\right|_{W_{2}}$, exactly one pebble can be moved to $R_{i+2}$ under $\left.D\right|_{W_{2}}$

(a) The vertex of $R_{i+2}$ used in $\left(R_{i+2}, v_{i+1} x \longrightarrow v_{i+2} x\right)$ is $v_{i+3} x$; then, if it continues, the rubbling move must be $\left(v_{i+3} y, v_{i+2} x \longrightarrow v_{i+2} y\right)$, but then we can use $\left(v_{i+1} y, v_{i+3} y \longrightarrow v_{i+2} y\right)$ instead, and thus we must use the strict rubbling move $\left(R_{i-1}, R_{i+1} \longrightarrow R_{i}\right)$ to solve at most one vertex $v_{i+2} x$. Let $P_{\text {left }}=v_{i+3}, \ldots, v_{n}$; then, the vertices of $P_{\text {left }} \times P_{2}$ are reachable without the strict rubbling moves and the pebble on $v_{i+1} y$. So, $|D|_{W_{2}} \mid-1 \geq \rho_{\text {opt }}\left(P_{\text {left }} \times P_{2}\right)$, and by induction, $\rho_{\text {opt }}\left(P_{\text {left }} \times P_{2}\right)+1=\rho_{\text {opt }}\left(W_{2}\right)$, over.

(b) The vertex of $R_{i+2}$ used in $\left(R_{i+2}, v_{i+1} x \longrightarrow v_{i+2} x\right)$ is $v_{i+2} y$; since at most one pebble can be moved to $R_{i+2}$, we may assume $D\left(v_{i+2} y\right)=1$ (if not, then we can move two pebbles to $v_{i+3} y$, and then one pebble can be moved to $v_{i+3} x$; this is just (a)). Thus, we use one pebble each on $R_{i-1}, v_{i+1} y$, and $v_{i+2} y$ to move one pebble to $v_{i+2} x$. Then, we rearrange the distribution on $P_{n} \times P_{2}$ as follows: remove the pebble on $v_{i+1} y$, and add it on $v_{i+2} y$; one can view that we can still solve $R_{i}$ and $R_{i+1}$. Thus, in the new distribution, we do not need the strict rubbling move $\left(R_{i-1}, R_{i+1} \longrightarrow R_{i}\right) \quad$ to $\quad$ solve $\quad W_{2}$, so $|D|_{W_{2}} \mid \geq \rho_{\text {opt }}\left(W_{2}\right)$.

Thus, $\rho_{\text {opt }}\left(W_{1}\right)+\rho_{\text {opt }}\left(W_{2}\right)=\rho_{\text {opt }}\left(H_{i-1}\right)+\rho_{\text {opt }}\left(H_{n-i-1}\right)$.

Assume that $n=3 k+r$ and $i=3 j+s$ for $0 \leq r, s \leq 2$; by induction, we can get Table 1, where the lower bound is given by $\rho_{\text {opt }}\left(H_{i-1}\right)+\rho_{\text {opt }}\left(H_{n-i-1}\right)$.

From Table 1 , we can find $|D| \geq f_{\text {opt }}\left(L_{n+1}\right)$, which is a contradiction to the assumption that $\rho_{\text {opt }}\left(P_{n} \times P_{2}\right)<f_{\text {opt }}\left(L_{n+1}\right)$, and we are done.

Case 2. Now we consider $H_{3 k+2}$. Note that the diameter of $H_{3 k+2}$ is $3 k+3$. Let $R_{i}$ be the vertices set $\left\{v_{i} x, v_{i} y\right\}$ for $1 \leq i \leq 3 k+2$, and $R_{3 k+3}=\left\{v_{3 k+3} y\right\}$. If we collapse $R_{i}$ into one vertex $r_{i}$ for $1 \leq i \leq 3 k+3$, then we can get a path $\widetilde{P}_{3 k+3}=$ $r_{1} \ldots r_{3 k+3}$ (see Figure 2). By Lemma 10, the optimal pebbling number of $\widetilde{P}_{3 k+3}$ is $f_{\text {opt }}\left(\widetilde{P}_{3 k+3}\right)=\lceil 2(3 k+3) / 3\rceil=2 k+2$. So, we only need to show that $\rho_{\text {opt }}\left(H_{3 k+2}\right)>f_{\text {opt }}\left(\widetilde{P}_{3 k+3}\right)$.

Let $D$ be a solvable distribution with $\rho_{\text {opt }}\left(H_{3 k+2}\right)$ pebbles on $H_{3 k+2}$ and $D^{*}$ be the quotient distribution on $\widetilde{P}_{3 k+3}$. So, $D^{*}$ is solvable on $\widetilde{P}_{3 k+3}$ by Lemma 8 .

Subcase 3. If $\rho_{\text {opt }}\left(H_{3 k+2}\right)<f_{\text {opt }}\left(\widetilde{P}_{3 k+3}\right)$, since $D^{*}$ is a solvable distribution on $\widetilde{P}_{3 k+3}$, there must exist some vertex $r_{i}$ of $\widetilde{P}_{3 k+3}$ which can be reachable Assume that $W_{1}$ and $W_{2}$ be two disjoint subgraphs of $H_{\{3 k+2\}}$ which induce $Q_{1}=r_{1}, \ldots$, $r_{\{i-1\}}$ and $Q_{2}=r_{\{i+1\}}, \ldots, r_{\{3 k+3\}}$, respectively.
We will show that $\left.D\right|_{W_{j}}$ is $W_{j}$-solvable for $j=1,2$. Otherwise, we must use the strict rubbling move $\left(R_{i-1}, R_{i+1} \longrightarrow R_{i}\right)$ under $D$ to solve one vertex of $W_{i}$; this move must be one of $\left(v_{i-1} x, v_{i+1} x \longrightarrow v_{i} x\right)$ and $\left(v_{i-1} y, v_{i+1} y \longrightarrow v_{i} y\right)$. Without loss of generality, assume the rubbling move is $\left(v_{i-1} x, v_{i+1} x \longrightarrow v_{i} x\right)$; then, by Lemma 2 , the target vertex of the next rubbling move using the pebble on $v_{i} x$ cannot be $v_{i+1} x$ or $v_{i-1} x$; if the target vertex is $v_{i} y$, we need one more pebble on $R_{i-1}$ or $R_{i+1}$, a contradiction to the condition that $r_{i}$ can be reachable only by the strict rubbling move $\left(r_{i-1}, r_{i+1} \longrightarrow r_{i}\right)$.

So, $\left.D\right|_{W_{i}}$ is $W_{i}$-solvable, and thus $|D|_{W_{i}} \mid \geq \rho_{\text {opt }}\left(W_{i}\right)$ for $i=1,2$. Note that $W_{1}$ is isomorphic to $P_{i-1} \times P_{2}$ and $W_{2}$ is isomorphic to $H_{3 k+2-i}$. Thus, $|D| \geq \rho_{\text {opt }}\left(P_{i-1} \times P_{2}\right)+\rho_{\text {opt }}\left(H_{3 k+2-i}\right)$. Assume that $i=3 j+s$ for $0 \leq s \leq 2$; by induction, we can get Table 2 , where the lower bound is given by $\rho_{\text {opt }}\left(P_{i-1} \times P_{2}\right)+\rho_{\text {opt }}\left(H_{3 k+2-i}\right)$.

From Table 2, we can find $\rho_{\text {opt }}\left(H_{3 k+2}\right)>f_{\text {opt }}\left(L_{3 k+3}\right)$, a contradiction to the assumption $\rho_{\text {opt }}\left(H_{3 k+2}\right)<f_{\text {opt }}\left(L_{3 k+3}\right)$, over.

Subcase 4. If $\rho_{\text {opt }}\left(H_{3 k+2}\right)=f_{\text {opt }}\left(L_{3 k+3}\right)=2 k+2$, then $D^{*}$ is solvable on $\widetilde{P}_{3 k+3}$. If there exists a vertex $r_{i}$ which is reachable only by a strict rubbling move under $D^{*}$, then we can get a contradiction by a similar argument of Subcase 3 .

So, we only need to consider the case that all vertices of $\widetilde{P}_{3 k+3}$ are reachable by only pebbling moves. By Lemma 11 , we have $D^{*}\left(R_{3 i+2}\right)=2$ for $0 \leq i \leq k$, and $D^{*}(v)=0$ otherwise. Thus, at least one of $\left\{v_{1} x, v_{1} y\right\}$ is not solvable under $D$, which completes the proof.

Theorem 4 (see [6]). The optimal rubbling number of prism $C_{n} \times P_{2}$ is

$$
\rho_{\mathrm{opt}}\left(C_{3 k+r} \times P_{2}\right)= \begin{cases}2 k, & \text { if } r=0, \\ 2 k+1, & \text { if } r=1=\left\lceil\frac{2 n}{3}\right\rceil, \\ 2 k+2, & \text { if } r=2,\end{cases}
$$

except $\rho_{\text {opt }}\left(C_{3} \times P_{2}\right)=3$.

Proof. It holds for $n=3$; we only need to show $\rho_{\text {opt }}\left(C_{n} \times\right.$ $\left.P_{2}\right)=\rho_{\text {opt }}\left(P_{n-1} \times P_{2}\right)$ for $n \geq 4$.

Upper Bound. $P_{n-1} \times P_{2}$ can be viewed as a subgraph of $C_{n} \times P_{2}$; it is clear that a solvable distribution with $\rho_{\text {opt }}\left(P_{n-1} \times P_{2}\right)$ pebbles given in the proof of Theorem 3 is solvable on $C_{n} \times P_{2}$ for $n \geq 4$, and we are done.

Lower Bound. Let $C_{n}=v_{1}, \ldots, v_{n}, P_{2}=x y$. Assume $D$ is a solvable distribution with $\rho_{\text {opt }}\left(C_{n} \times P_{2}\right)$ pebbles, and we collapse each set $\left\{v_{i} x, v_{i} y\right\}$ into one vertex $R_{i}$ (see Figure 3); then, we get a cycle $C_{n}=R_{1}, \ldots, R_{n}$ and the quotient distribution $D^{*}$. By Lemma $8, D^{*}$ is solvable on $C_{n}$. By Lemma $10, f_{\text {opt }}\left(C_{n}\right)=\lceil 2 n / 3\rceil$, and we only need to show $\rho_{\text {opt }}\left(C_{n} \times P_{2}\right)=f_{\text {opt }}\left(C_{n}\right)$.

Assume $\rho_{\text {opt }}\left(C_{n} \times P_{2}\right)<f_{\text {opt }}\left(C_{n}\right)$; then, there must exist a vertex $R_{i}$ which is reachable under $D^{*}$ only by the strict rubbling move $\left(R_{i-1}, R_{i+1} \longrightarrow R_{i}\right)$. By a similar argument of 
TABle 1: Bounds on optimal rubbling of $P_{n} \times P_{2}$.

\begin{tabular}{lccccccccc}
\hline$(r, s)$ & $(0,0)$ & $(0,1)$ & $(0,2)$ & $(1,0)$ & $(1,1)$ & $(1,2)$ & $(2,0)$ & $(2,1)$ & $(2,2)$ \\
\hline$\rho_{\text {opt }}\left(H_{i-1}\right)$ & $2 j+1$ & $2 j+1$ & $2 j+2$ & $2 j+1$ & $2 j+1$ & $2 j+2$ & $2 j+1$ & $2 j+1$ & $2 j+2$ \\
$\rho_{\text {opt }}\left(H_{n-i-1}\right)$ & $2(k-j)+1$ & $2(k-j)$ & $2(k-j)-1$ & $2(k-j)+1$ & $2(k-j)+1$ & $2(k-j)$ & $2(k-j)+2$ & $2(k-j)+1$ & $2(k-j)+1$ \\
Lower bound & $2 k+2$ & $2 k+1$ & $2 k+1$ & $2 k+2$ & $2 k+2$ & $2 k+2$ & $2 k+3$ & $2 k+2$ & $2 k+3$ \\
$f_{\text {opt }}\left(L_{n+1}\right)$ & $2 k+1$ & $2 k+1$ & $2 k+1$ & $2 k+2$ & $2 k+2$ & $2 k+2$ & $2 k+2$ & $2 k+2$ & $2 k+2$ \\
\hline
\end{tabular}

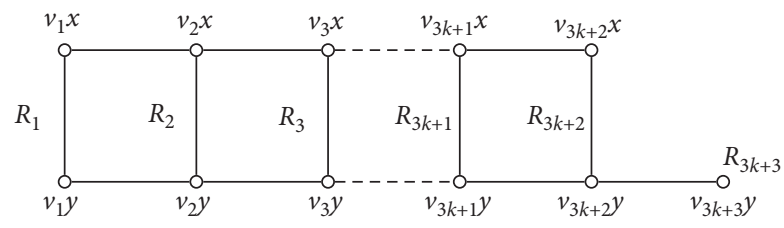

Figure 2: $H_{3 k+2}$.

TABLE 2: Bounds on optimal rubbling of $H_{3 k+2}$.

\begin{tabular}{lccc}
\hline$s$ & 0 & 1 & 2 \\
\hline$\rho_{\text {opt }}\left(P_{i-1} \times P_{2}\right)$ & $2 j+1$ & $2 j+2$ & $2 j+2$ \\
$\rho_{\text {opt }}\left(H_{3 k+2-i}\right)$ & $2(k-j)+2$ & $2(k-j)+1$ & $2(k-j)+1$ \\
Lower bound & $2 k+3$ & $2 k+3$ & $2 k+3$ \\
$f_{\text {opt }}\left(L_{3 k+3}\right)$ & $2 k+2$ & $2 k+2$ & $2 k+2$ \\
\hline
\end{tabular}

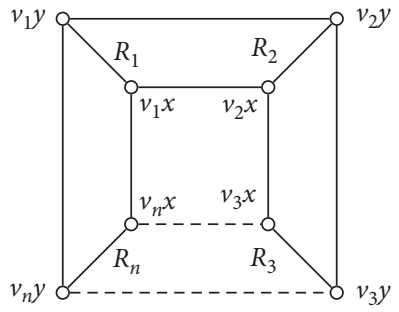

Figure 3: $C_{n} \times P_{2}$.

Case 3 in the proof of Theorem 3 , if we remove $\left\{v_{i} x, v_{i} y\right\}$ from $C_{n} \times P_{2}$ (which is just isomorphic to $P_{n-1} \times P_{2}$ ), then $D$ is still solvable. So, $|D| \geq \rho_{\text {opt }}\left(P_{n-1} \times P_{2}\right)=\lceil 2 n / 3\rceil$, which is a contradiction to the assumption $\rho_{\text {opt }}\left(C_{n} \times P_{2}\right)<f_{\text {opt }}\left(C_{n}\right)$, and we are done.

Theorem 5 (see [6]). The optimal rubbling number of the Möbius ladder $M_{n}$ is

$$
\rho_{\text {opt }}\left(M_{n}\right)=\rho_{\text {opt }}\left(C_{n} \times P_{2}\right) \text {. }
$$

Proof. The proof is similar to the proof of prisms.

\section{Bounds on the Optimal Rubbling of $P_{m} \times P_{n}$}

In [12], the optimal pebbling number of $P_{3} \times P_{n}$ is determined.

Theorem 6 (see [12]). The optimal pebbling number of $P_{3} \times$ $P_{n}$ is $n+1$.

In [13], the lower bound of the optimal pebbling number of $P_{m} \times P_{n}$ is given.
Theorem 7 (see [13]). The optimal pebbling number of $P_{m} \times$ $P_{n}$ is no less than (2/13)mn when $m, n \geq 5$.

Here we give an upper bound of the optimal rubbling number of $P_{m} \times P_{n}$ by giving a solvable distribution on $P_{m} \times P_{n}$.

Theorem 8. $\rho_{\text {opt }}\left(P_{3} \times P_{n}\right) \leq\lceil 5 n+1 / 6\rceil+1$.

Proof. We give a solvable distribution $D_{n}$ on $P_{3} \times P_{n}$ with exactly $\lceil 5 n+1 / 6\rceil+1$ pebbles. While $n \leq 7$, the solvable distribution $D_{n}$ is given in Figure 4 .

While $n>7$, assume $n \equiv r(\bmod 6)$ for $0 \leq r \leq 5$. Then, the solvable distribution $D_{n}$ on $P_{3} \times P_{n}$ can be obtained from $D_{n-6}$ on $P_{3} \times P_{n-6}$ while $r \neq 1$ by placing five more pebbles on $P_{3} \times P_{6}$ which is added on the left side of $P_{3} \times P_{n-6}$ (see Figure $5(\mathrm{a}))$. So, $\left|D_{n}\right|=\left|D_{n-6}\right|+5$ for $n \equiv r(\bmod 6)$ with $r \neq q 1$.

Assume $n>7$ and $n \equiv 1(\bmod 6)$. Then, the solvable distribution $D_{n}$ on $P_{3} \times P_{n}$ can be obtained from $D_{n-5}$ on $P_{3} \times P_{n-5}$ by placing four more pebbles on $P_{3} \times P_{5}$ which is added on the left side of $P_{3} \times P_{n-5}$ (see Figure 5(b)). So, $\left|D_{n}\right|=\left|D_{n-5}\right|+4$ for $n \equiv 1(\bmod 6)$. 


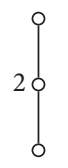

(a)

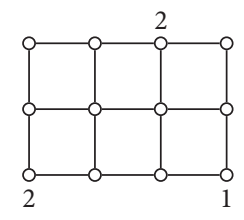

(d)

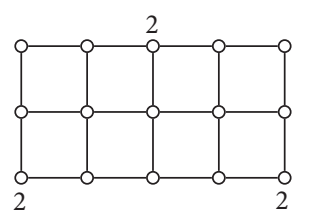

(e)

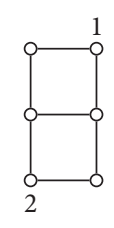

(b)

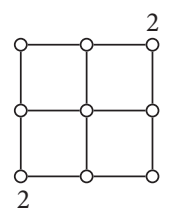

(c)

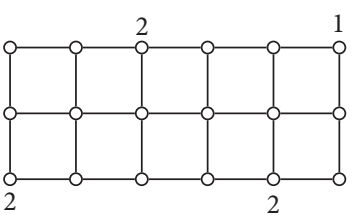

(f)

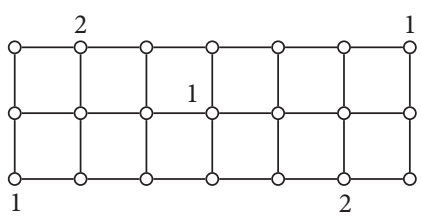

(g)

Figure 4: A solvable distribution $D_{n}$ on $P_{3} \times P_{n}$ for $n \leq 7$.

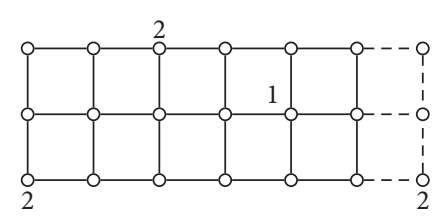

(a)

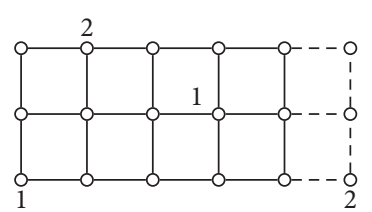

(b)

Figure 5: A solvable distribution $D_{n}$ from $D_{n-6}$ or $D_{n-5}$. (a) $D_{n}$ from $D_{n-6}$. (b) $D_{n}$ from $D_{n-5}$.

By a simple calculation, we have $\left|D_{n}\right|=\lceil 5 n+1 / 6\rceil+1$, and we are done.

One can check that the equality holds for $n \leq 7$, and here we give a conjecture.

Conjecture 1. $\rho_{\text {opt }}\left(P_{3} \times P_{n}\right)=\lceil 5 n+1 / 6\rceil+1$.

Now we can get a bound of the optimal rubbling number of $P_{m} \times P_{n}$. We give a partition of $P_{m}$, for example, $m=x+1+y$; then, we can get three disjoint subgraphs of $P_{m} \times P_{n} \quad$ called $H_{1}=P_{x} \times P_{n}, \quad H_{2}=P_{1} \times P_{n} \quad$ and $H_{3}=P_{y} \times P_{n}$, and then we put some pebbles on $H_{1}$ and $H_{3}$, so that the pebbles on $H_{i}$ can solve $H_{i}$ for $i=1,3$. Clearly, this is a solvable distribution of $P_{m} \times P_{n}$. Thus, we have the following.

Theorem 9. $\rho_{\text {opt }}\left(P_{4} \times P_{n}\right) \leq \rho_{\text {opt }}\left(P_{n}\right)+\rho_{\text {opt }}\left(P_{2} \times P_{n}\right)$, $\rho_{\text {opt }}\left(P_{5} \times P_{n}\right) \leq \rho_{\text {opt }}\left(P_{2} \times P_{n}\right)+\rho_{\text {opt }}\left(P_{2} \times P_{n}\right)$.

Theorem 10. If $m=4 k+r \geq 6, \quad \rho_{\text {opt }}\left(P_{4 k} \times P_{n}\right) \leq$ $(k-1) \rho_{\text {opt }}\left(P_{3} \times \quad P_{n}\right)+\rho_{\text {opt }}\left(P_{2} \times P_{n}\right)+\rho_{\text {opt }}\left(P_{n}\right)$, $\rho_{\text {opt }}\left(P_{4 k+1} \times P_{n}\right) \leq(k-1) \rho_{\text {opt }}\left(P_{3} \times P_{n}\right)+2 \rho_{\text {opt }}\left(P_{2} \times P_{n}\right)$, $\rho_{\text {opt }}\left(P_{4 k+2} \times P_{n}\right) \leq k \rho_{\text {opt }}\left(P_{3} \times P_{n}\right)+\rho_{\text {opt }}\left(P_{2} \times P_{n}\right)$, $\rho_{\text {opt }}\left(P_{4 k+3} \times P_{n}\right) \leq(k+1) \rho_{\text {opt }}\left(P_{3} \times P_{n}\right)$.

Proof. If $m=4 k$, then $m=3+1+3+1+\cdots+3+1+2+$ $1+1$ which gives a partition of $P_{m}=P_{3}+P_{1}+P_{3}+P_{1}+\cdots+P_{3}+P_{1}+P_{2}+P_{1}+P_{1}$. We put the pebbles on $P_{i} \times P_{n}$ except the inner subpaths $P_{1} \times P_{n}$, so that the pebbles on each $P_{i} \times P_{n}$ can solve themselves, and thus we need $(k-1) \rho_{\text {opt }}\left(P_{3} \times P_{n}\right)+\rho_{\text {opt }}\left(P_{2} \times P_{n}\right)+\rho_{\text {opt }}\left(P_{n}\right) \quad$ pebbles, which is a solvable distribution. Similarly, we can get the other inequalities, which completes the proof.

$\begin{array}{llll}\text { Corollary } & \text { 1. If } & 6 \leq m \leq n, & \text { then }\end{array}$ $\rho_{\text {opt }}\left(P_{m} \times P_{n}\right) \leq(5 / 24) m n+(1 / 3) n+(1 / 2) m+(1 / 2)$.

Proof. From Theorems 2, 3, 8, and 10, we can get the result by a simple calculation.

\section{Data Availability}

No data were used to support the findings of the study.

\section{Disclosure}

This manuscript has been released as a pre-print at https:// arxiv.org/pdf/1909.01703v1.pdf.

\section{Conflicts of Interest}

The authors declare that they have no conflicts of interest regarding the publication of the article.

\section{Acknowledgments}

This research was supported by Key Projects in Natural Science Research of Anhui Provincial Department of Education (nos. KJ2018A0438 (to Zheng-Jiang Xia) and KJ2020A0015 (to Zhen-Mu Hong)) and NSFC (no. 11601002 (to Zhen-Mu Hong)). 


\section{References}

[1] F. R. K. Chung, "Pebbling in hypercubes," SIAM Journal on Discrete Mathematics, vol. 2, no. 4, pp. 467-472, 1989.

[2] G. Hurlbert, "General graph pebbling," Discrete Applied Mathematics, vol. 161, no. 9, pp. 1221-1231, 2013.

[3] C. Belford and N. Sieben, "Rubbling and optimal rubbling of graphs," Discrete Mathematics, vol. 309, no. 10, pp. 3436-3446, 2009.

[4] L. Danz, Optimal t-rubbling of Complete m-ary Trees, University of Minnesota Duluth, Duluth, MN, USA, 2010.

[5] F. László, Optimal Rubbling Numbers of Graphs (In Hungarian), Budapest University of Technology and Economics, Budapest, Hungary, 2010.

[6] G. Y. Katona and L. F. Papp, "The optimal rubbling number of ladders, prisms and Möbius-ladders," Discrete Applied Mathematics, vol. 209, pp. 227-246, 2016.

[7] G. Y. Katona and N. Sieben, "Bounds on the rubbling and optimal rubbling numbers of graphs," Electronic Notes in Discrete Mathematics, vol. 38, pp. 487-492, 2011.

[8] B. Crull, T. Cundiff, P. Feltman et al., "The cover pebbling number of graphs," Discrete Mathematics, vol. 296, no. 1, pp. 15-23, 2005.

[9] D. P. Bunde, E. W. Chambers, D. Cranston, K. Milans, and D. B. West, "Pebbling and optimal pebbling in graphs," Journal of Graph Theory, vol. 57, no. 3, pp. 215-238, 2008.

[10] D. Hersovici, "Grahams conjecture on products of cycles," Journal of Graph Theory, vol. 42, pp. 141-154, 2003.

[11] L. Pachter, H. S. Snevily, and B. Voxman, "On pebbling graphs,” The Combinatorics Numer.vol. 107, pp. 65-80, 1995.

[12] C. Xue and C. Yerger, "Optimal pebbling on grids," Graphs and Combinatorics, vol. 32, no. 3, pp. 1229-1247, 2016.

[13] E. Győri, G. Y. Katona, and L. F. Papp, "Optimal pebbling number of the square grid," Graphs and Combinatorics, vol. 36, pp. 803-829, 2020. 